Article

\title{
Prediction of Weld Reinforcement Based on Vision Sensing in GMA Additive Manufacturing Process
}

\author{
Rongwei Yu®, Zhuang Zhao *, Lianfa Bai and Jing Han * \\ Jiangsu Key Laboratory of Spectral Imaging and Intelligent Sense, Nanjing University of Science and Technology, \\ Nanjing 210094, China; yrw_njust@163.com (R.Y.); blf@njust.edu.cn (L.B.) \\ * Correspondence: zhaozhuang3126@gmail.com (Z.Z.); eohj@njust.edu.cn (J.H.); Tel.: +86-151-9599-5329 (Z.Z. \& J.H.)
}

Received: 25 June 2020; Accepted: 29 July 2020; Published: 2 August 2020

\begin{abstract}
In the gas-metal-arc (GMA) additive manufacturing process, the shape of the molten pool, the temperature field of the workpiece and the heat dissipation conditions change with the increase of cladding layers, which can affect the dimensional accuracy of the workpiece; hence, it is necessary to monitor the additive manufacturing process online. At present, there is little research about formation-dimension monitoring in the GMA additive manufacturing process; in this paper, weld reinforcement prediction in the GMA additive manufacturing process was conducted, the visual-sensing system for molten pool was established, and a laser locating system was designed to match every frame of the molten pool image with the actual weld location. Extracting the shape and location features of the molten pool as visual features, on the basis of a back-propagation (BP) neural network, we developed the prediction model for weld reinforcement in the GMA additive manufacturing process. Experiment results showed that the model could accurately predict weld reinforcement. By changing the cooling time between adjacent cladding layers, the generalization ability of the prediction model was further verified.
\end{abstract}

Keywords: GMA additive manufacturing; weld reinforcement; visual features; neural network

\section{Introduction}

Additive manufacturing technology is based on the idea of discreteness and accumulation, using material to stack layer by layer, forming a three-dimensional entity [1]. In recent years, using additive manufacturing technology to obtain a workpiece with high dimensional accuracy and good mechanical properties has become a research hotspot [2]. Gas-metal-arc (GMA) additive manufacturing is the processing technology that uses an electric arc as a heat source to melt metal wire and stacks, forming a metal workpiece [3,4], Yanhu Wang et al. [5,6] pointed out that additive manufacturing based on arc welding has the outstanding advantages of low cost and high efficiency, and can be widely used in many fields. They studied the additive manufacturing of copper-aluminum alloys by adding a small amount of silicon in the cold-metal-transfer (CMT) welding process. In recent years, the visual-sensing method has been widely used in the field of welding manufacturing, Zhuang Zhao et al. [7] proposed an optimal imaging-band selection mechanism for molten pool vision, which has important guiding significance for collecting high-quality molten pool images. Jun Lu et al. [8] obtained the temperature field of the molten pool on the basis of visual imaging in the GMA welding process, and realized the prediction of hump weld bead by monitoring the temperature field distribution of the molten pool.

In the GMA additive manufacturing process, with the increase of cladding layers, heat accumulation of the workpiece is serious, heat dissipation worsens and the shape of the molten pool changes, which ultimately affect the dimensional accuracy of the formed workpiece, so it is very important to monitor the GMA additive manufacturing process online [9]. Ouyang et al. [10,11] produced aluminum-alloy workpieces in a variable-polarity gas-tungsten-arc-welding (GTAW) additive manufacturing process. 
They used a charge coupled device (CCD) to monitor the arc length online, pointing out that arc length and preheating temperature have important influence on forming quality. Spencer et al. [12] produced a metal workpiece in a GMA additive manufacturing process, using an infrared thermometer to monitor online the temperature of the welding workpiece. When the temperature of the welding workpiece cooled to a certain value, the next layer could be stacked. This method reduces the time efficiency of the additive manufacturing process, but improves the microstructure and material properties. Kwak et al. $[13,14]$ used two CCD sets to monitor the morphology of the cladding layer in the metal-inert-gas (MIG) welding additive manufacturing process, and used an infrared thermographer to detect the surface-temperature field of the welding workpiece. Jinqian Zhu et al. [15] used an infrared thermographer to detect online the temperature field of the cladding channel in a laser metal-wire additive manufacturing process, realized the prediction of the cladding channel width and the accurate location of welding defects.

The artificial-neural-network model has excellent nonlinear-mapping characteristics, as well as strong learning and induction abilities; the support vector machine (SVM) is a classifier with sparsity and robustness. In recent years, they have been widely used in areas of pattern recognition, and fault diagnosis, prediction, and estimation. Yukang Liu et al. [16] developed a dynamic adaptive neural fuzzy inference system in a GTAW process, and predicted the weld width on the reverse side of the welding seam online by using the visual characteristic parameters of the molten pool. Yanfeng Gao et al. [17,18] developed an SVM to predict the welding-penetration mode through the arc sound signal during the welding process. Jiajia Yang et al. [19] acquired a molten pool image in the near-infrared band in an aluminum-alloy double-wire-welding process, extracted the visual features of molten pool, and established the penetration-state recognition model on the basis of a neural network.

At present, research on GMA additive manufacturing mainly focuses on the feasibility of the forming process, and the mechanical properties of the formed workpiece [20,21]; there is little research on the dimensional accuracy of the formed workpiece [9]. In this paper, we took single-pass multilayer GMA additive manufacturing as the research object, used a color CCD to collect molten pool images and extract the shape and position features of the molten pool in real time; by means of a neural network, established the prediction model for weld reinforcement, and realized the online monitoring of weld reinforcement in the additive manufacturing process, which has important guiding significance for the subsequent control of forming size.

\section{Welding Experiment Platform and Approach}

\subsection{Welding Experiment Platform}

Conducting arc additive manufacturing experiment in the CMT welding procedure, which belongs to the GMA welding procedure, the welding experiment platform included a welding power supply (Fronius CMT advanced 4000R), a mobile robot (ABB IRB1400 M2004, ABB, Zurich, Switzerland), a molten pool visual sensor, and a laser locating system, which are shown in Figure 1 . The base metal was 304 stainless steel plate, the size was $450 \mathrm{~mm} \times 150 \mathrm{~mm} \times 10 \mathrm{~mm}$, and the welding wire was stainless steel, the diameter of which was $1.2 \mathrm{~mm}$, the chemical composition of welding wire is shown in Table 1, the type of welding torch was Robacta Drive CMT (Pettenbach, Austria). The visual sensor for the molten pool consisted of a color camera (Basler acA640-750uc, Ahrensburg, Germany), computer, and trigger module; the color camera was installed on the welding torch. Furthermore, we developed a laser locating system that consisted of a laser, monochrome camera (Basler ace acA1920-155um, Ahrensburg, Germany), and computer; the laser was installed on the welding torch, and sent out light shining on the upper edge of welding wire. The monochrome camera was fixed on the experiment platform to record the moving trajectory of the laser point. In the GMA additive manufacturing process, the trigger module gave out a fixed frequency signal to control the color and monochrome cameras at the same time; by observing the location of the laser point in every frame of the monochrome image, precisely matching every frame of color molten pool images with the specific weld location. 


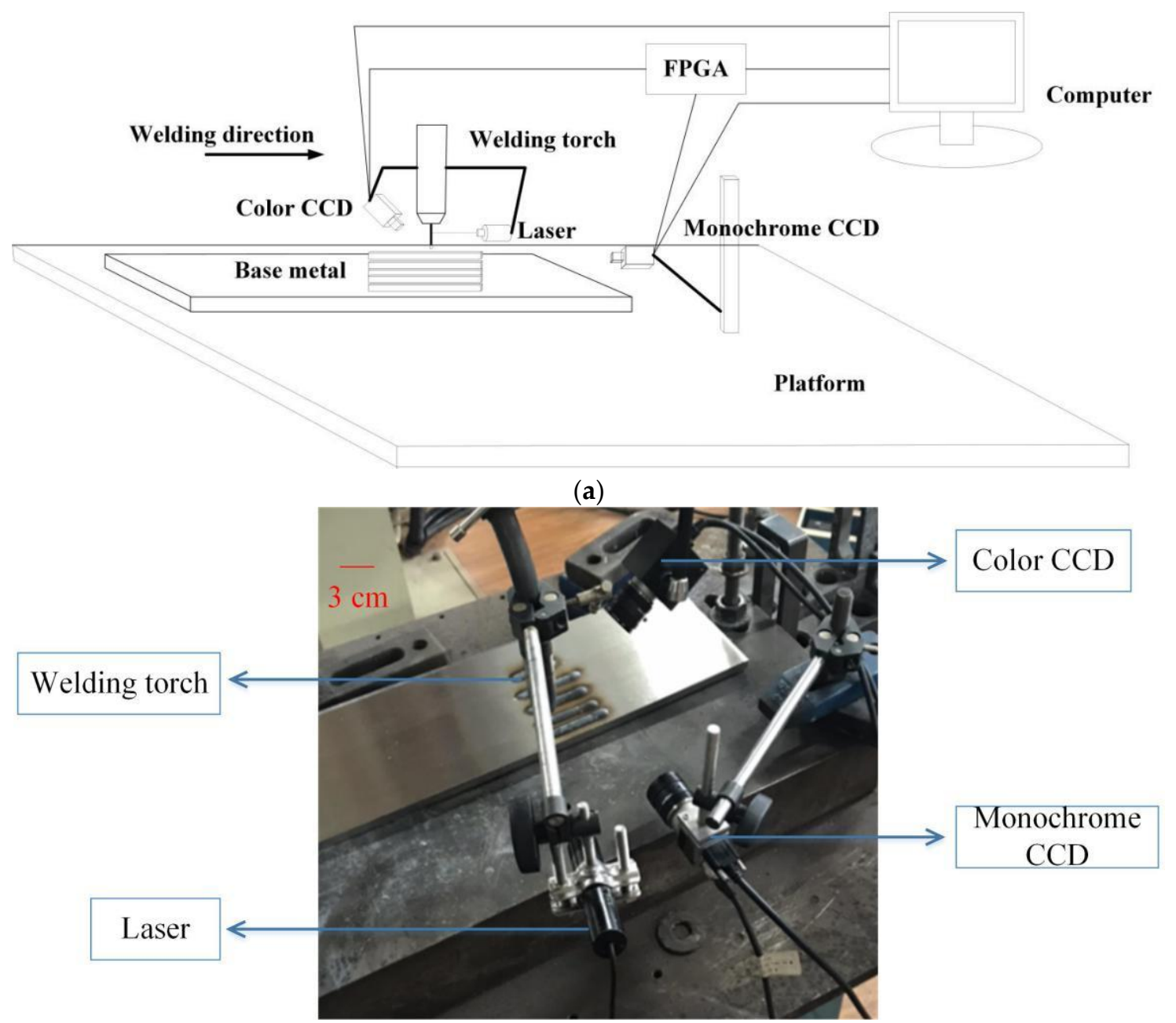

(b)

Figure 1. Welding experiment platform. (a) Structure diagram; (b) Physical diagram.

Table 1. The chemical composition of welding wire.

\begin{tabular}{ccccccc}
\hline Elements & $\mathbf{C}$ & $\mathbf{M n}$ & $\mathbf{C r}$ & $\mathbf{N i}$ & $\mathbf{M o}$ & $\mathbf{S i}$ \\
\hline Composition & $0.012 \%$ & $2.180 \%$ & $18.97 \%$ & $12.70 \%$ & $2.310 \%$ & $0.570 \%$ \\
\hline
\end{tabular}

\subsection{Experiment Approach}

In the GMA additive manufacturing experiment, the welding torch moved horizontally and in the same direction, setting the contact tip to work distance (CTWD) to $1.5 \mathrm{~cm}$, welding current to $130 \mathrm{~A}$, the welding velocity to $5 \mathrm{~mm} / \mathrm{s}$, the welding length of each cladding layer to $80 \mathrm{~mm}$, and the number of cladding layers to 10; detailed parameters are shown in Table 2. In the GMA additive manufacturing process, the trigger module gave out fixed frequency signal to control the color and monochrome cameras at the same time, whose frequency was $1000 \mathrm{~Hz}$. We extracted the shape and location features of the molten pool through color molten pool image. By observing the location of the laser point in every frame of the monochrome image, we determined the actual weld location corresponding to each frame collected color molten pool images. After each cladding layer was welded, using three-dimensional scanner to measure the height of the workpiece, we extracted the weld reinforcement. All extracted data constituted the data set of the prediction model. 
Table 2. Welding parameters.

\begin{tabular}{ccccc}
\hline Welding Current & Welding Voltage & Welding Speed & Wire Feeding Speed & Shield Gas Flux \\
\hline $129 \mathrm{~A}$ & $14.4 \mathrm{~V}$ & $5 \mathrm{~mm} / \mathrm{s}$ & $4.9 \mathrm{~m} / \mathrm{min}$ & $25 \mathrm{~L} / \mathrm{min}$ \\
\hline
\end{tabular}

\section{Experiment Data Extraction}

\subsection{Definition and Extraction of Visual Feature Parameters}

In the GMA additive manufacturing process, after the color CCD had completed molten pool image acquisition, binary processing was carried out to extract the molten pool outline. Holes were filled up in the contour, and the shape features of the molten pool could be determined. We calculated the molten pool area, length, and width, which were selected as the shape features. Molten pool area was defined as whole pixels within the molten pool outline, molten pool length was defined as the maximal distance of the molten pool contour along the welding direction, and molten pool width was defined as the maximal distance of the molten pool contour perpendicular to the welding direction.

At the arc-striking stage, the cooling rate of the molten pool was relatively fast, the molten pool was difficult to spread out. With the increase of cladding layers, the workpiece tended to be bogged down, which is shown in Figure 2; in addition, the welding procedure is an important factor. The geometric dimensions of the workpiece are shown in Figure 3. The relative position of the molten pool in the image would change; therefore, the location feature of the molten pool is also an important feature. We extracted the wire extension as the location feature of the molten pool. Figure 4 is the schematic diagram of the visual features of the molten pool.

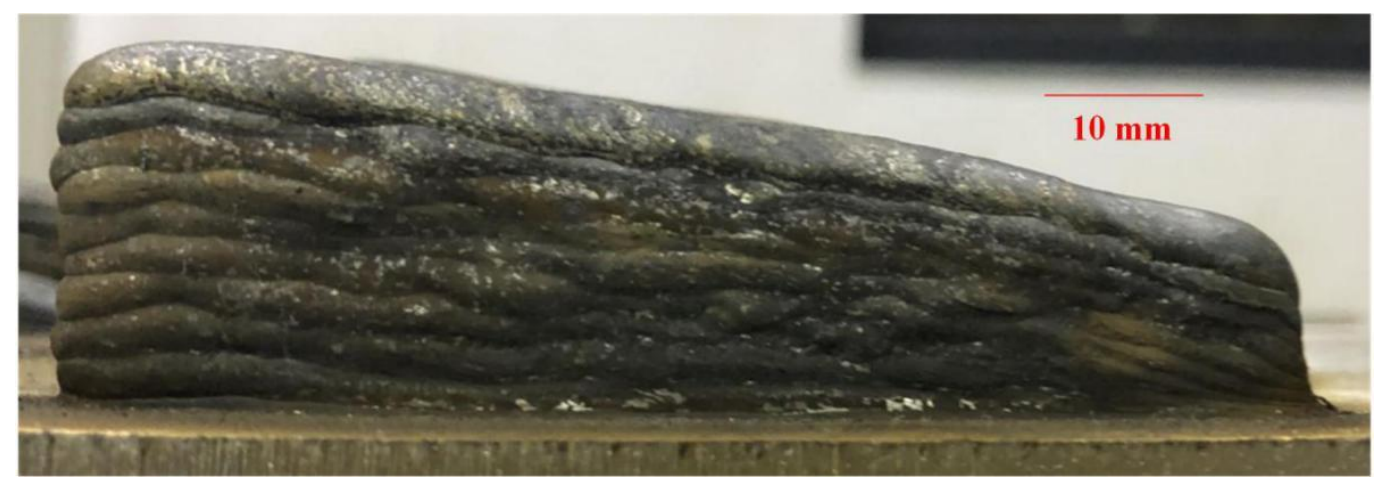

Figure 2. Workpiece formed by additive manufacturing.

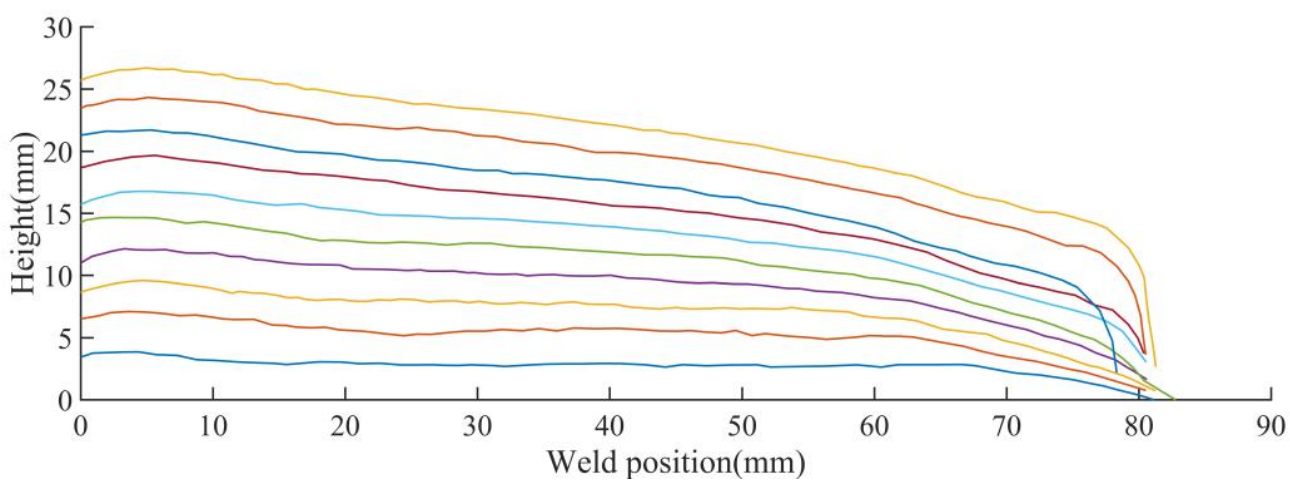

Figure 3. The geometric dimensions of the workpiece. 


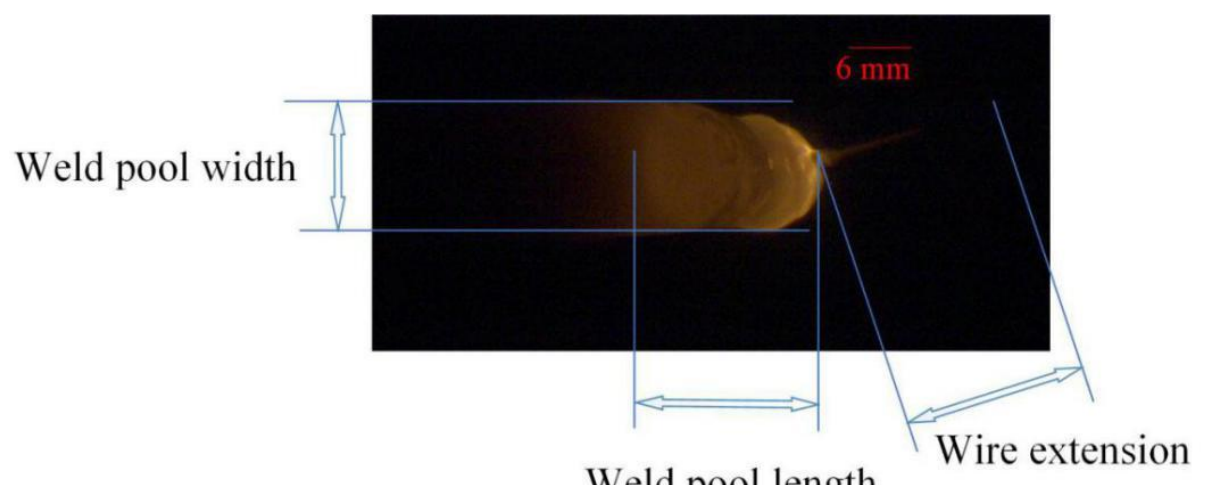

Figure 4. Schematic diagram of visual features of molten pool.

In this paper, the additive manufacturing experiment was conducted in the CMT procedure. The advantages of the CMT welding process are small splash, low heat input, and stable arc. After setting the welding parameters, the current waveform was collected in the actual welding process. Figure 5 [22] shows the welding current waveform of the CMT process under certain welding parameters, while, Figure 6 [22] shows all images collected by color CCD within a CMT cycle.

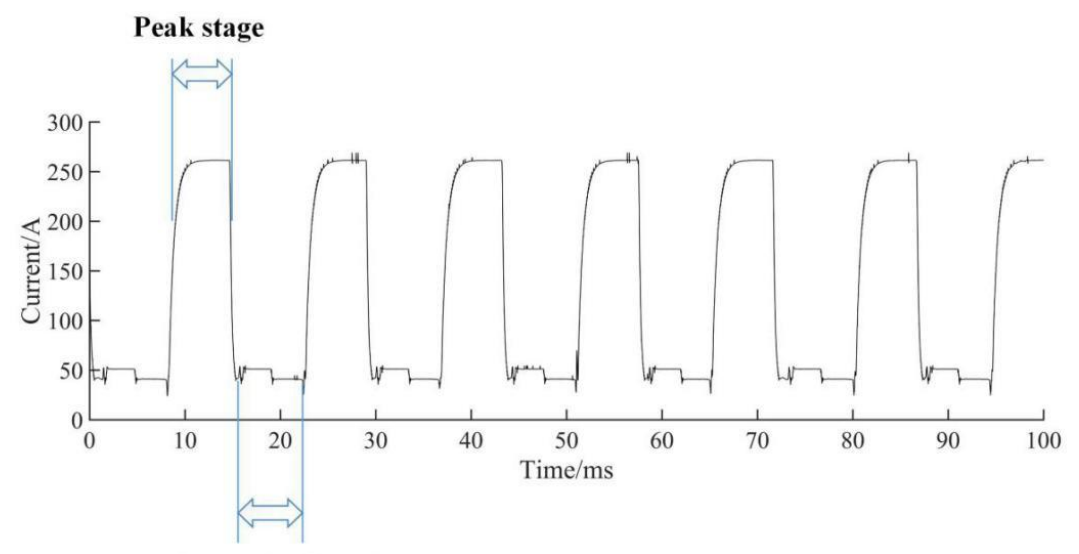

Arc extinction stage

Figure 5. Welding current waveform of cold-metal-transfer (CMT) procedure.

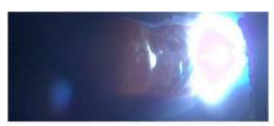

$1 \mathrm{~ms}$

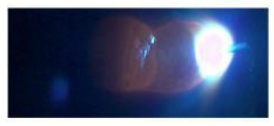

$5 \mathrm{~ms}$

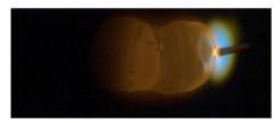

$9 \mathrm{~ms}$

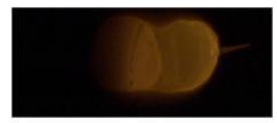

$13 \mathrm{~ms}$

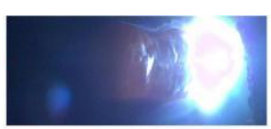

$2 \mathrm{~ms}$

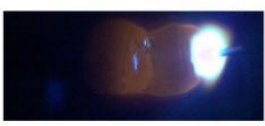

$6 \mathrm{~ms}$

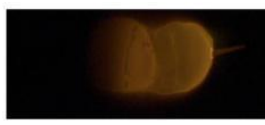

$10 \mathrm{~ms}$

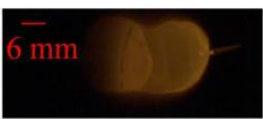

$14 \mathrm{~ms}$

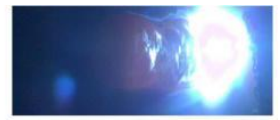

$3 \mathrm{~ms}$

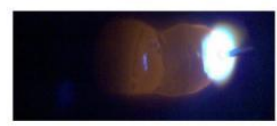

$7 \mathrm{~ms}$

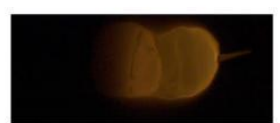

$11 \mathrm{~ms}$

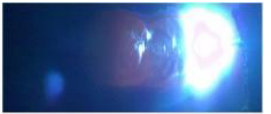

$4 \mathrm{~ms}$

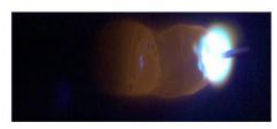

$8 \mathrm{~ms}$

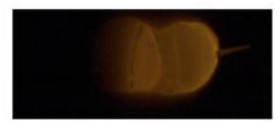

$12 \mathrm{~ms}$

Figure 6. All images collected within CMT cycle. 
Figures 5 and 6 show that the CMT cycle was approximately $14 \mathrm{~ms}$, at the peak stage of CMT process. Because of the strong arc light, the collected molten pool image was seriously disturbed, and it was difficult to calculate the visual features of the molten pool. At the base stage of the CMT process, there was almost no arc interference, and the collected molten pool image had high signal-to-noise ratio (SNR), from which the shape and location features of the molten pool could be accurately extracted. In this paper, we chose the first frame image collected at the base stage within every CMT cycle, and extracted the shape and location features of the molten pool.

\subsection{Extraction of Weld Reinforcement}

In the GMA additive manufacturing process, after each cladding layer was welded, using a 3D scanner (Wiiboox REEYEE 3M) to measure the height of the workpiece (the 3D scanner is shown in Figure 7), getting the relationship between workpiece height and welding seam position, the height difference between adjacent cladding layers was defined as the weld reinforcement of the current cladding layer. Then, by means of the laser locating system, we could calculate the weld reinforcement corresponding to every frame of the molten pool image collected by color CCD.

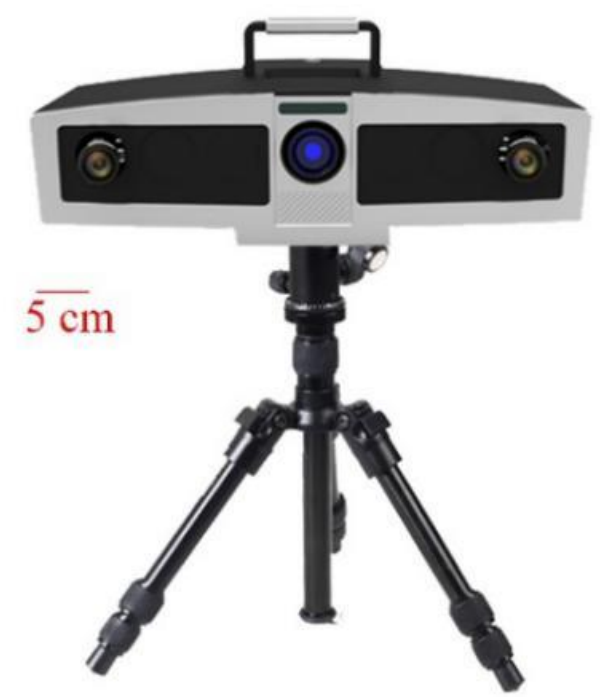

Figure 7. Three-dimensional scanner.

\section{Prediction-Model Establishment for Weld Reinforcement}

Artificial neural networks have excellent nonlinear mapping characteristics and strong learning ability. In recent years, they have developed rapidly and are widely used in automatic control, prediction, and other fields. Back-propagation (BP) neural networks are multilayer feedforward neural networks that are trained by error back-propagation algorithms.

Taking the shape and location features of the current frame molten pool as inputs, that is to say, the molten pool area, length, width, and the wire extension as inputs, the weld reinforcement corresponding to the current frame as the output; by means of the BP neural network, the prediction model for weld reinforcement was developed. There were two hidden layers in the neural network. On the basis of the test results, two hidden layers were set with 10 neurons, respectively; the structure of the prediction model is shown in Figure 8. 


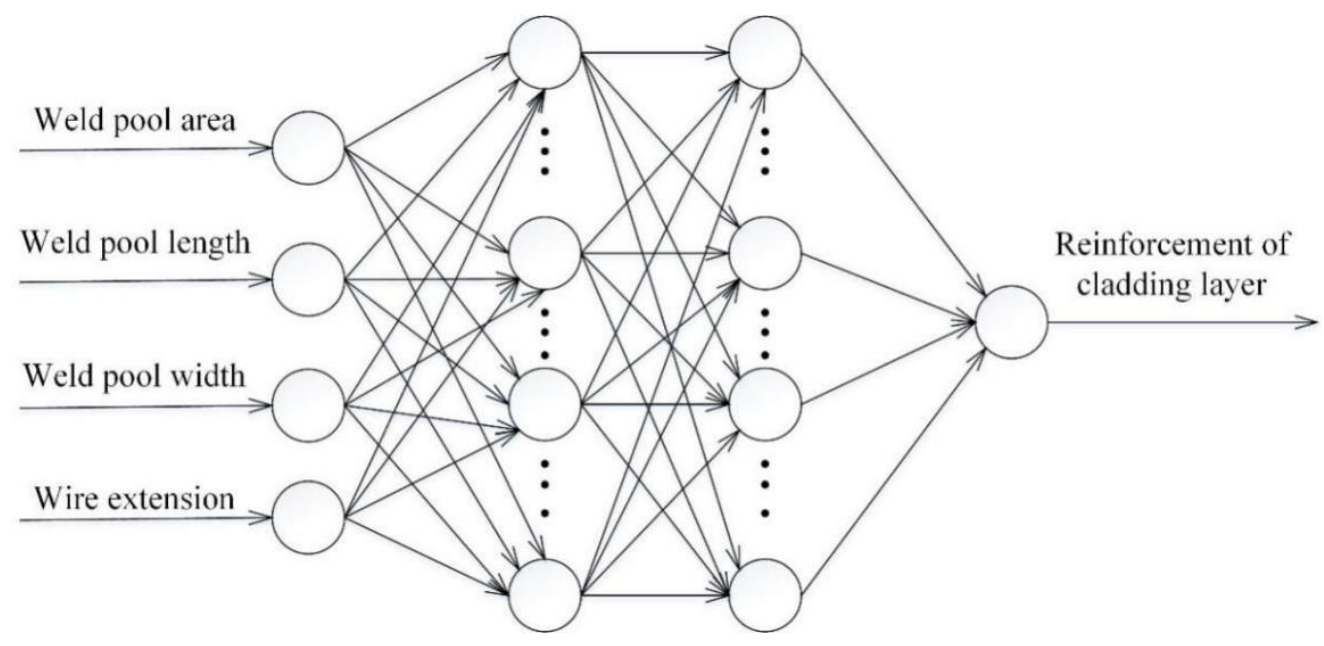

Figure 8. Structure of prediction model.

In the GMA additive manufacturing process, accumulating one layer every $3 \mathrm{~min}$, that is to say, the cooling time between adjacent cladding layers was $3 \mathrm{~min}$, according to the acquired data, we randomly selected 800 groups of data to constitute the training set in each cladding layer, and the other data constituted the test set; detailed data groups are shown in Table 3. Partial data of the shape and location features of the molten pool, and the corresponding weld reinforcement are shown in Table 4 .

Table 3. Number of data groups within training and test sets.

\begin{tabular}{ccc}
\hline Number of Cladding Layers & Training Set & Test Set \\
\hline 3 & 800 & 129 \\
4 & 800 & 142 \\
5 & 800 & 169 \\
6 & 800 & 129 \\
7 & 800 & 145 \\
8 & 800 & 165 \\
9 & 800 & 139 \\
10 & 800 & 54 \\
\hline
\end{tabular}

Table 4. Partial data of molten pool features and corresponding weld reinforcement.

\begin{tabular}{ccccc}
\hline $\begin{array}{c}\text { Molten Pool Area } \\
\text { (pixel) }\end{array}$ & $\begin{array}{c}\text { Molten Pool } \\
\text { Length (pixel) }\end{array}$ & $\begin{array}{c}\text { Molten Pool } \\
\text { Width (pixel) }\end{array}$ & $\begin{array}{c}\text { Wire Extension } \\
\text { (pixel) }\end{array}$ & $\begin{array}{c}\text { Weld Reinforcement } \\
\text { (mm) }\end{array}$ \\
\hline 19440 & 171 & 146 & 44 & 2.1551 \\
25037 & 212 & 155 & 58 & 2.2781 \\
24453 & 217 & 155 & 58 & 1.9829 \\
22752 & 210 & 140 & 62 & 2.0542 \\
21025 & 198 & 129 & 68 & 1.7656 \\
20227 & 183 & 139 & 76 & 1.8179 \\
19228 & 181 & 131 & 84 & 1.6907 \\
16954 & 152 & 137 & 108 & 1.5000 \\
16036 & 149 & 136 & 131 & 1.7075 \\
\hline
\end{tabular}

Before training and testing the prediction model, all data needed to be mapped to [0,1], that is normalization, and the detailed calculation formula is:

$$
x_{n e w}=\frac{x_{\text {origin }}-x_{\min }}{x_{\max }-x_{\min }}
$$


where $x_{\text {origin }}$ and $x_{\text {new }}$ are the numerical values before and after treatment, respectively; and $x_{\max }$ and $x_{\min }$ are the maximal and minimal numerical values within the original data set, respectively.

\section{Predicted Results and Analysis}

By testing the established prediction model, the predicted results of weld reinforcement in the third, fourth, and fifth cladding layers are shown in Figure 9.

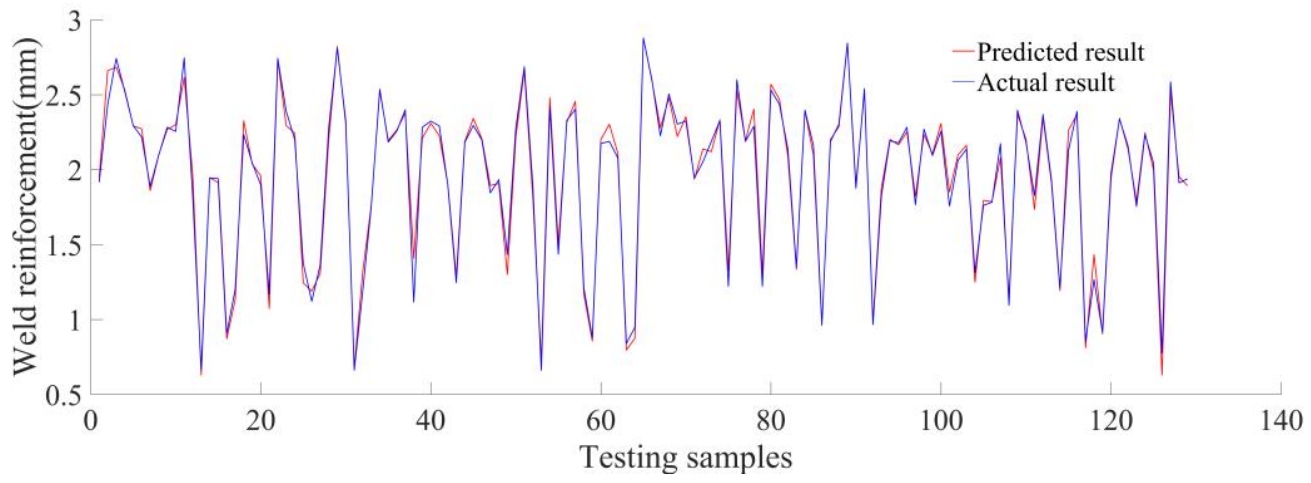

(a)

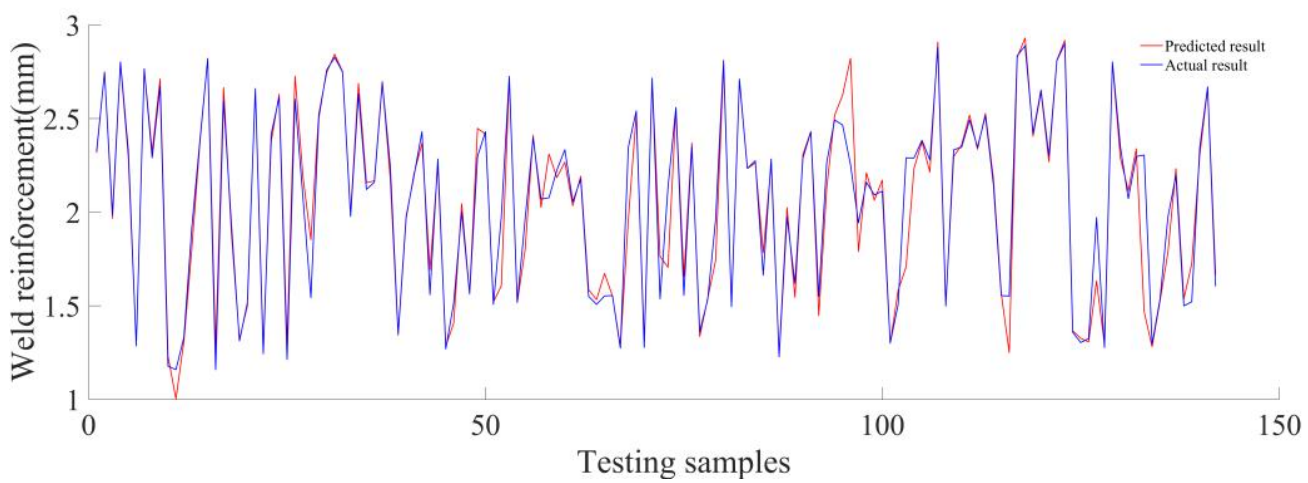

(b)

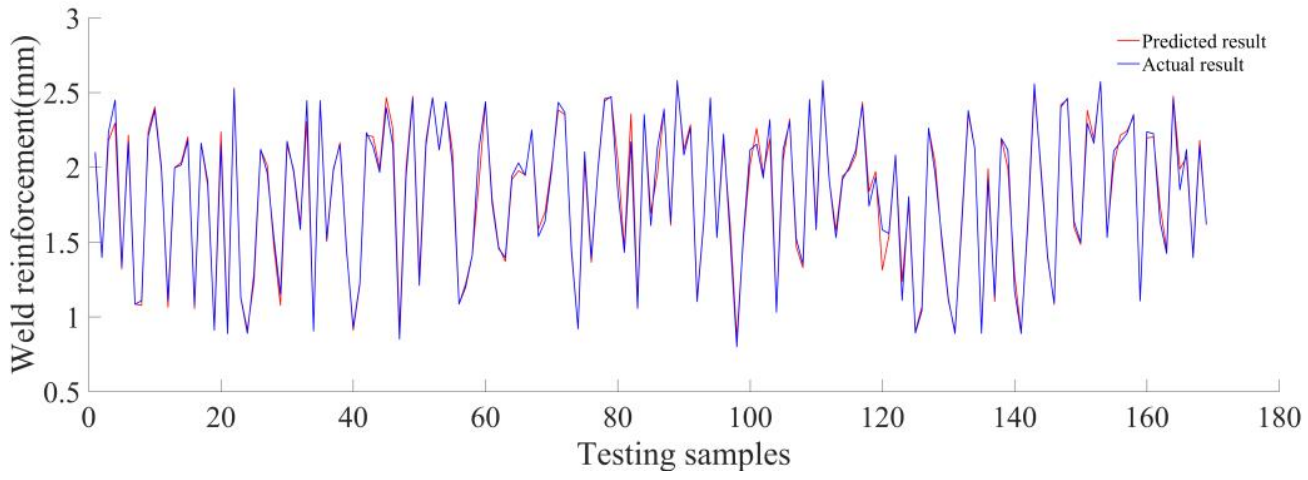

(c)

Figure 9. Predicted results of weld reinforcement. (a) Third cladding layer; (b) Fourth cladding layer; (c) Fifth cladding layer.

There are several reasons for the larger prediction error of individual samples, for example, the welding process is a non-linear and multivariable process, and there are some random uncertainties, which leads to the deviation of individual experiment results from the predicted results of the prediction model. In addition, there were some errors in the extraction and calculation of visual features of the molten pool. 
The predicted precision of the developed model could be judged through mean absolute error $(M A E)$; the calculation equation is as follows:

$$
M A E=\frac{1}{m} \cdot \sum_{i=1}^{m}\left|h^{\prime}(i)-h(i)\right|
$$

where $h^{\prime}$ denotes the predicted weld reinforcement, $h$ denotes the actual weld reinforcement, and $m$ is the whole number of data groups within the test set.

The predicted precision of the established prediction model is shown in Table 5.

Table 5. Predicted precision of established model.

\begin{tabular}{cccc}
\hline Number of Cladding Layers & MAE $(\mathbf{m m})$ & Number of Cladding Layers & MAE (mm) \\
\hline 3 & 0.0476 & 7 & 0.0412 \\
4 & 0.0699 & 8 & 0.0431 \\
5 & 0.0388 & 9 & 0.0439 \\
6 & 0.0313 & 10 & 0.0353 \\
\hline
\end{tabular}

In the GMA additive manufacturing process, if cooling time between adjacent cladding layers is kept constant, the weld reinforcement of each cladding layer greatly fluctuates; the established prediction model in this paper could accurately predict weld reinforcement. In the GMA additive manufacturing process, the heat-dissipation condition in each cladding layer, and the change rule of the molten pool shape are different; therefore, there is no necessary relationship between MAE and the ordinal number of cladding layers. By reducing the number of data groups in the training set, the test accuracy of the model did not decrease, which indicated that the selected number of data groups in the training set was enough.

When all the data acquired from different cladding layers were combined into one data group, randomly selecting 150 groups of data as the test set, and the other data constituted the training set, the predicted results of weld reinforcement are shown in Figure 10; predicted MAE was 0.1094 mm.

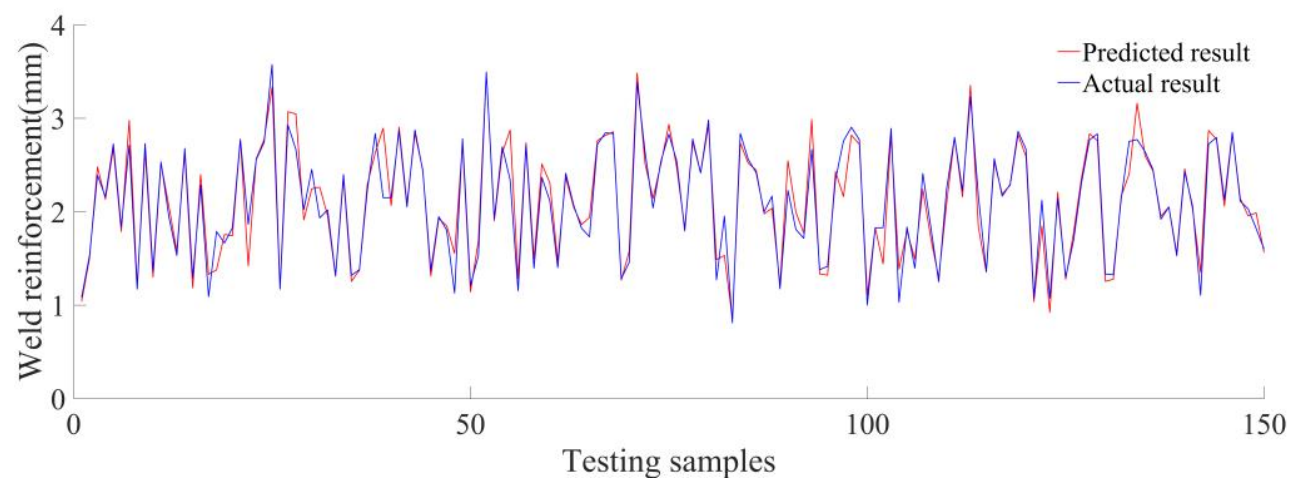

Figure 10. Predicted results of weld reinforcement.

\section{Verification of Generalization Ability of Prediction Model}

In the GMA additive manufacturing process, cooling time between adjacent cladding layers has great influence on the shape of the molten pool and the precision of the final forming dimension. In order to further verify the generalization ability of the prediction model for weld reinforcement, we changed the cooling time between adjacent cladding layers, with other welding parameters remaining unchanged.

In the case of cooling time between adjacent cladding layers being $4 \mathrm{~min}$, according to the acquired data, we randomly selected 800 groups of data to constitute the training set in each cladding 
layer, and the other data constituted the test set, as shown in Table 6. The predicted precision of the established prediction model is shown in Table 7.

Table 6. Number of data groups within training and test sets.

\begin{tabular}{ccc}
\hline Number of Cladding Layers & Training Set & Test Set \\
\hline 3 & 800 & 152 \\
4 & 800 & 148 \\
5 & 800 & 186 \\
6 & 800 & 141 \\
7 & 800 & 150 \\
8 & 800 & 163 \\
9 & 800 & 155 \\
10 & 800 & 145 \\
\hline
\end{tabular}

Table 7. Predicted precision of the established model.

\begin{tabular}{cccc}
\hline Number of Cladding Layers & MAE $(\mathbf{m m})$ & Number of Cladding Layers & MAE $(\mathbf{m m})$ \\
\hline 3 & 0.0312 & 7 & 0.0335 \\
4 & 0.0320 & 8 & 0.0304 \\
5 & 0.0358 & 9 & 0.0348 \\
6 & 0.0228 & 10 & 0.0583 \\
\hline
\end{tabular}

When all data acquired from different cladding layers were combined into one data group, randomly selecting 150 groups of data as the test set, and the other data constituted the training set, the predicted results of weld reinforcement are shown in Figure 11; predicted MAE was $0.0777 \mathrm{~mm}$.

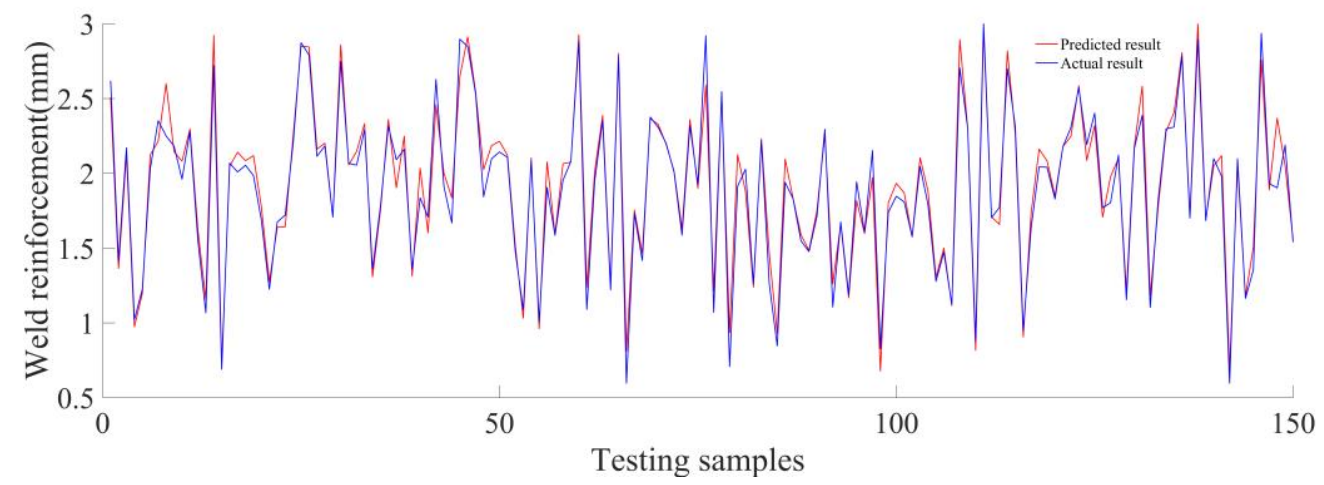

Figure 11. Predicted results of weld reinforcement.

In the case of cooling time between adjacent cladding layers being $2 \mathrm{~min}$, according to the acquired data, we randomly selected 800 groups of data to constitute the training set in each cladding layer, and the other data constituted the test set, as shown in Table 8. The predicted precision of the established prediction model is shown in Table 9. 
Table 8. Number of data groups within training and test sets.

\begin{tabular}{ccc}
\hline Number of Cladding Layers & Training Set & Test Set \\
\hline 3 & 800 & 112 \\
4 & 800 & 188 \\
5 & 800 & 145 \\
6 & 800 & 129 \\
7 & 800 & 113 \\
8 & 800 & 144 \\
9 & 800 & 111 \\
10 & 800 & 108 \\
\hline
\end{tabular}

Table 9. Predicted precision of established model.

\begin{tabular}{cccc}
\hline Number of Cladding Layers & MAE $(\mathbf{m m})$ & Number of Cladding Layers & MAE $(\mathbf{m m})$ \\
\hline 3 & 0.0352 & 7 & 0.0549 \\
4 & 0.0473 & 8 & 0.0353 \\
5 & 0.0365 & 9 & 0.0391 \\
6 & 0.0274 & 10 & 0.0563 \\
\hline
\end{tabular}

When all data acquired from different cladding layers were combined into one data group, randomly selecting 150 groups of data as the test set, and the other data constituted the training set, the predicted results of weld reinforcement are shown in Figure 12; predicted MAE was $0.0950 \mathrm{~mm}$.

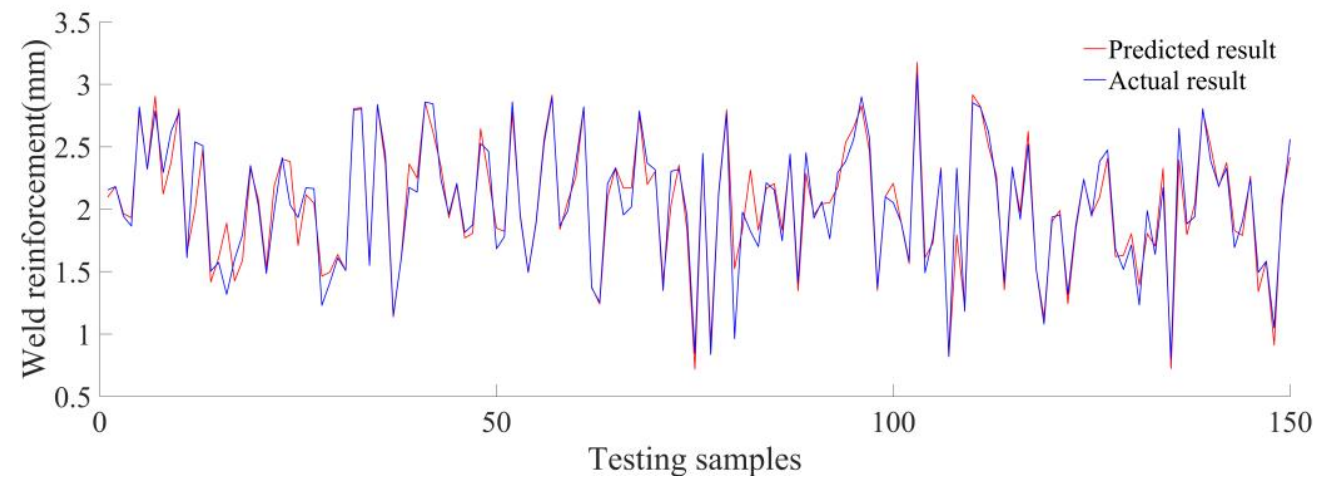

Figure 12. Predicted results of weld reinforcement.

Similarly, due to different heat-dissipation conditions in each cladding layer, the change rule of the molten pool shape was also different; there was no necessary relationship between MAE value and the cooling time of adjacent cladding layers. To sum up, we can conclude that the prediction model for weld reinforcement had high prediction precision and strong generalization ability, which could be applied to the GMA additive manufacturing process.

\section{Conclusions}

(1) A vision-sensing system for molten pool was established that can extract the shape feature of molten pool in real time. A laser positioning system was also developed to match every frame collected molten pool images with actual weld location.

(2) Taking the shape and location features of the current frame molten pool as input, the weld reinforcement corresponding to current frame as the output, by means of a BP neural network, the prediction model for weld reinforcement was developed; experiment results showed that the predicted MAE of the model was less than $0.11 \mathrm{~mm}$. By changing cooling time between adjacent cladding layers, the generalization ability of prediction model was further verified. 
(3) Future work will mainly focus on the study of the online control method for weld reinforcement in the GMA additive manufacturing process.

Author Contributions: Conceptualization, methodology, writing-original draft, R.Y.; investigation, writing-review and editing, Z.Z.; supervision, L.B. and J.H. All authors have read and agreed to the published version of the manuscript.

Funding: This work was supported by the National Natural Science Foundation of China (61727802 and 61901220), the Key Research and Development programs in Jiangsu China (BE2018126), and the Jiangsu postdoctoral research funding program (2019K216).

Conflicts of Interest: The authors declare no conflict of interest.

\section{References}

1. Lu, B.-H.; Li, D.-C. Development of the Additive Manufacturing (3D printing) Technology. Mach. Build. Autom. 2013, 42, 1-4.

2. Xiong, J.; Zhang, G. Adaptive control of deposited height in GMAW-based layer additive manufacturing. J. Mater. Process. Technol. 2014, 214, 962-968. [CrossRef]

3. Mughal, M.P.; Fawad, H.; Mufti, R.A. Three-Dimensional Finite-Element Modelling of Deformation in Weld-Based Rapid Prototyping. Proc. Inst. Mech. Eng. Part C J. Mech. Eng. Sci. 2006, 220, 875-885. [CrossRef]

4. Kazanas, P.; Deherkar, P.; De Almeida, P.M.; Lockett, H.; Williams, S. Fabrication of geometrical features using wire and arc additive manufacture. Proc. Inst. Mech. Eng. Part B J. Eng. Manuf. 2012, 226, 1042-1051. [CrossRef]

5. Wang, Y.; Chen, X.; Konovalov, S.V. Additive Manufacturing Based on Welding Arc: A low-Cost Method. J. Surf. Investig. X-ray Synchrotron Neutron Tech. 2017, 11, 1317-1328. [CrossRef]

6. Wang, Y.; Chen, X.; Konovalov, S.; Su, C.; Siddiquee, A.N.; Gangil, N. In-situ wire-feed additive manufacturing of Cu-Al alloy by addition of silicon. Appl. Surf. Sci. 2019, 487, 1366-1375. [CrossRef]

7. Zhao, Z.; Deng, L.; Bai, L.; Zhang, Y.; Han, J. Optimal imaging band selection mechanism of weld pool vision based on spectrum analysis. Opt. Laser Technol. 2019, 110, 145-151. [CrossRef]

8. Lu, J.; Zhao, Z.; Han, J.; Bai, L.-F. Hump weld bead monitoring based on transient temperature field of molten pool. Optik 2020, 208, 164031. [CrossRef]

9. Xiong, J.; Zhang, G. Online measurement of bead geometry in GMAW-based additive manufacturing using passive vision. Meas. Sci. Technol. 2013, 24, 115103. [CrossRef]

10. Wang, H.; Jiang, W.; Ouyang, J.; Kovacevic, R. Rapid prototyping of 4043 Al-alloy parts by VP-GTAW. J. Mater. Process. Technol. 2004, 148, 93-102. [CrossRef]

11. Ouyang, J.; Wang, H.; Kovacevic, R. Rapid prototyping of 5356-aluminum alloy based on variable polarity gas tungsten arc welding: Process control and microstructure. Mater. Manuf. Process. 2002, 17, 103-124. [CrossRef]

12. Spencer, J.D.; Dickens, P.M.; Wykes, C.M. Rapid prototyping of metal parts by three-dimensional welding. Proc. Inst. Mech. Eng. Part B J. Eng. Manuf. 1998, 212, 175-182. [CrossRef]

13. Kwak, Y.-M.; Doumanidis, C. Geometry Regulation of Material Deposition in Near-Net Shape Manufacturing by Thermally Scanned Welding. J. Manuf. Process. 2002, 4, 28-41. [CrossRef]

14. Doumanidis, C.; Kwak, Y.-M. Geometry Modeling and Control by Infrared and Laser Sensing in Thermal Manufacturing with Material Deposition. J. Manuf. Sci. Eng. 2000, 123, 45-52. [CrossRef]

15. Zhu, J.; Ling, Z.; Du, F.; Ding, X.; Li, H. Monitoring of laser metal-wire additive manufacturing temperature field using infrared thermography. Infrared Laser Eng. 2018, 47, 0604002.

16. Liu, Y.K.; Zhang, W.J.; Zhang, Y.M. Estimation of Weld Joint Penetration under Varying GTA Pools. Weld. J. 2013, 92, 313-321.

17. Gao, Y.; Zhao, J.; Wang, Q.; Xiao, J.; Zhang, H. Weld bead penetration identification based on human-welder subjective assessment on welding arc sound. Measurement 2020, 154, 107475. [CrossRef]

18. Wang, Q.; Gao, Y.; Huang, L.; Gong, Y.; Xiao, J. Weld bead penetration state recognition in GMAW process based on a central auditory perception model. Measurement 2019, 147, 106901. [CrossRef]

19. Yang, J.; Wang, K.; Wu, T.; Zhou, X. Welding penetration recognition in aluminum alloy tandem arc welding based on visual characters of weld pool. Trans. China Weld. Inst. 2017, 38, 49-52. 
20. Zhang, Y.M.; Chen, Y.; Li, P.; Male, A.T. Weld deposition-based rapid prototyping: A preliminary study. J. Mater. Process. Technol. 2003, 135, 347-357. [CrossRef]

21. Xiong, J.; Zhang, G.; Gao, H.; Wu, L. Modeling of bead section profile and overlapping beads with experimental validation for robotic GMAW-based rapid manufacturing. Robot. Comput. Manuf. 2013, 29, 417-423. [CrossRef]

22. Yu, R.; Han, J.; Zhao, Z.; Bai, L. Real-Time Prediction of Welding Penetration Mode and Depth Based on Visual Characteristics of Weld Pool in GMAW Process. IEEE Access 2020, 8, 81564-81573. [CrossRef]

(c) (C) 2020 by the authors. Licensee MDPI, Basel, Switzerland. This article is an open access article distributed under the terms and conditions of the Creative Commons Attribution (CC BY) license (http://creativecommons.org/licenses/by/4.0/). 